\title{
Transbronchial Biopsy Using Endobronchial Ultrasonography with a Guide Sheath Increased the Diagnostic Yield of Peripheral Pulmonary Lesions
}

\author{
Masayuki Ishida, Motoi Suzuki, Akitsugu Furumoto, Yoshiko Tsuchihashi, \\ Koya Ariyoshi and Konosuke Morimoto
}

\begin{abstract}
Objective The advantage of transbronchial biopsy (TBB) using endobronchial ultrasonography (EBUS) with a guide sheath (GS) over TBB without EBUS guidance was investigated in this study.

Materials and Methods A retrospective chart review was conducted at Nagasaki University Hospital, Japan. Data were collected from all cases of peripheral pulmonary lesions (PPLs) undergoing either EBUS-GSguided TBB or TBB without EBUS guidance in our department from December 2003 through November 2009. The diagnostic yield in each group was compared, after adjustment for other factors.

Results In total 110 PPLs were investigated in 102 patients: 65 (59.1\%) were examined with EBUS-GSguided TBB (EBUS-GS group) and 45 (40.9\%) were TBB without EBUS guidance (non-EBUS group). Both procedures were performed under $\mathrm{x}$-ray fluoroscopy. Basic characteristics were similar between the two groups. Of all EBUS examined lesions, $53(81.5 \%)$ were visualized by EBUS. The diagnostic yields in EBUS-GS group and non-EBUS group were $64.6 \%$ and $46.7 \%$, respectively $(\mathrm{p}=0.08)$. Adjusting for size and location of lesions, the yield of EBUS-GS guidance was 1.46 (95\% confidence interval 1.03 to 2.05) times higher than without EBUS guidance. When the lesion was visualized by EBUS, the diagnostic yield ratio was further increased to 1.63 (95\% CI 1.16 to 2.27).

Conclusion EBUS-GS-guided TBB demonstrates a higher diagnostic yield than TBB without EBUS guidance.
\end{abstract}

Key words: diagnostic yield, endobronchial ultrasonography, guide sheath, peripheral pulmonary lesions, solitary pulmonary nodule, transbronchial biopsy

(Intern Med 51: 455-460, 2012)

(DOI: 10.2169/internalmedicine.51.6358)

\section{Introduction}

Flexible bronchoscopic transbronchial biopsy (TBB) has been typically performed under $\mathrm{x}$-ray fluoroscopic guidance to sample peripheral pulmonary lesions (PPLs) in Japan. While the role of this procedure has been well established (1), its major limitation is that small nodules are difficult or impossible to visualize by conventional $\mathrm{x}$-ray fluoroscopy $(2,3)$. According to a meta-analysis, the diagnostic yield of fluoroscopy-guided TBB for nodules of $<20 \mathrm{~mm}$ diameter was only $33 \%$ (1).
Endobronchial ultrasonography (EBUS) with a radial probe was developed to improve yield. Studies have shown that many fluoroscopically invisible lesions are localized by EBUS images (3). The diagnostic yield of EBUS-guided TBB on solitary pulmonary nodules (SPNs) of $<30 \mathrm{~mm}$ diameter may reach $80 \%$ (4-8). In a randomized controlled study, EBUS guidance had significant advantage in diagnosing small-sized lung cancers over fluoroscopic guidance without EBUS (5). However, despite its diagnostic effectiveness, EBUS has problems. The EBUS probe needs to be removed from the lesion before introducing the biopsy forceps, and consequently it can be difficult to confirm whether 
the forceps is being properly inserted into the target bronchus as indicated by EBUS examinations. This can limit the potential diagnostic usefulness of TBB using EBUS $(7,8)$.

In 2004, Kurimoto et al (8) reported a new technique of EBUS-guided TBB using a guide sheath (GS). Under EBUS-GS-guidance, the GS remains in the target bronchus after removing the EBUS probe, and the forceps can be introduced through it. Thus, the forceps is more likely to be inserted to the EBUS-determined bronchus than by the EBUS-alone technique. Studies have shown that EBUS-GSguided TBB achieves high diagnostic yields both in masses and nodules (8-11). Eberhardt et al (12) have recently reported that definitive diagnoses were established for $46 \%$ of SPNs of $<20 \mathrm{~mm}$ diameter by TBB using EBUS with GS.

These findings suggest that EBUS-GS-guidance is the most accurate method to collect samples from PPLs among currently available bronchoscopic procedures (7). Its high diagnostic yield may also prevent additional invasive procedures such as CT-guided needle biopsy (CT-NB) and surgical biopsy. However, to our knowledge, there have been no reports formally comparing the diagnostic yield of EBUSGS-guided TBB to that of conventional TBB without EBUS guidance.

In the present study, we conducted a retrospective chart review to evaluate the effect of EBUS-GS-guided TBB on the diagnostic yield of PPLs. We also compared the effect on yield by the size of lesions.

\section{Materials and Methods}

\section{Subjects}

This was a retrospective study, which targeted all cases of PPLs which had been investigated with either EBUS-GSguided TBB or TBB without EBUS guidance in our department from December 2003 through November 2009. We evaluated the diagnostic yield of these procedures on the PPLs.

\section{Study setting}

Nagasaki University Hospital is a tertiary referral teaching hospital in Nagasaki City, Japan. The Department of Clinical Medicine, Institute of Tropical Medicine, Nagasaki University has 20 beds at Nagasaki University Hospital and provides medical care for patients with respiratory diseases and infectious diseases.

\section{Transbronchial biopsy}

The EBUS-GS system was introduced to our hospital in March 2008. Before the introduction of EBUS-GS, all TBBs for collecting samples from PPLs were performed under fluoroscopy guidance. A flexible fiberoptic bronchoscope (mainly BF T200: Olympus Medical Systems; Tokyo, Japan) was inserted transorally under local anesthesia. After complete inspection of the bronchial trees, the target lesion was localized fluoroscopically. A flexible transbronchial forceps was then advanced to the lesion and one to five specimens were taken.

After the introduction of EBUS-GS, all PPLs were initially evaluated by EBUS and biopsy was attempted through a guide sheath (SG-200C; Olympus; external diameter $1.95 \mathrm{~mm}$ ) under $\mathrm{x}$-ray fluoroscopy. A 20-MHz radialtype probe (UM-S20-17S; Olympus) with an external diameter of $1.4 \mathrm{~mm}$ was inserted through GS in the bronchoscope working channel. The EBUS probe and GS were confirmed to reach the PPL by EBUS images. Once the lesion was visualized by EBUS, the probe was withdrawn from the GS. A biopsy forceps was introduced into the GS until the point marked by a marker to reach the distance determined by the EBUS probe. When the lesion was not visualized by EBUS, both probe and GS were removed and TBB was performed under fluoroscopic guidance as for the conventional technique. Bronchial washing and brushing were performed following TBB with or without EBUS guidance.

Lesions visible on bronchoscope were excluded from the study. All lesions by which TBB had been performed after EBUS examinations were included in the EBUS-GS group even if they were invisible by EBUS.

\section{Data collection and analysis}

We calculated that 43 cases per group (a total of 86 cases) were required to detect the diagnostic yield ratio of 1.8 (diagnostic yield of conventional method $=40 \%$ ) with $80 \%$ power and a two-sided 0.05 alpha-level test.

Three physicians (MI, MS and KM) reviewed electronically recorded medical charts and collected necessary data using standardized information sheet. When the collected specimens showed findings of carcinoma by histopathology or class IV or V by cytology, TBB was considered to have yielded the diagnosis. For benign diseases including infectious diseases and inflammatory lung diseases, TBB was considered to have yielded diagnoses if the histological, cytological, or bacteriological findings were compatible with subsequent clinical outcomes.

Data were entered into the electronic database developed on EpiData Entry 3.1 (the EpiData Association, Denmark). The dataset was transferred to STATA 10 (Stata Corp., USA) for all statistical analyses. To compare the basic characteristics of EBUS-GS-guided TBB group (EBUS-GS group) and TBB without EBUS guidance group (non-EBUS group), chi-squared tests for categorical variables and Wilcoxon rank sum tests for continuous variables were performed. To compare the diagnostic yields between two groups, unadjusted and adjusted diagnostic yield ratios were calculated using Poisson regression models (13). All statistical tests were two-tailed and performed at an a priori alphalevel of 0.05 .

\section{Ethics}

This study was approved by the Institutional Review Board of the Institute of Tropical Medicine, Nagasaki University, Japan. Written informed consent was obtained from 


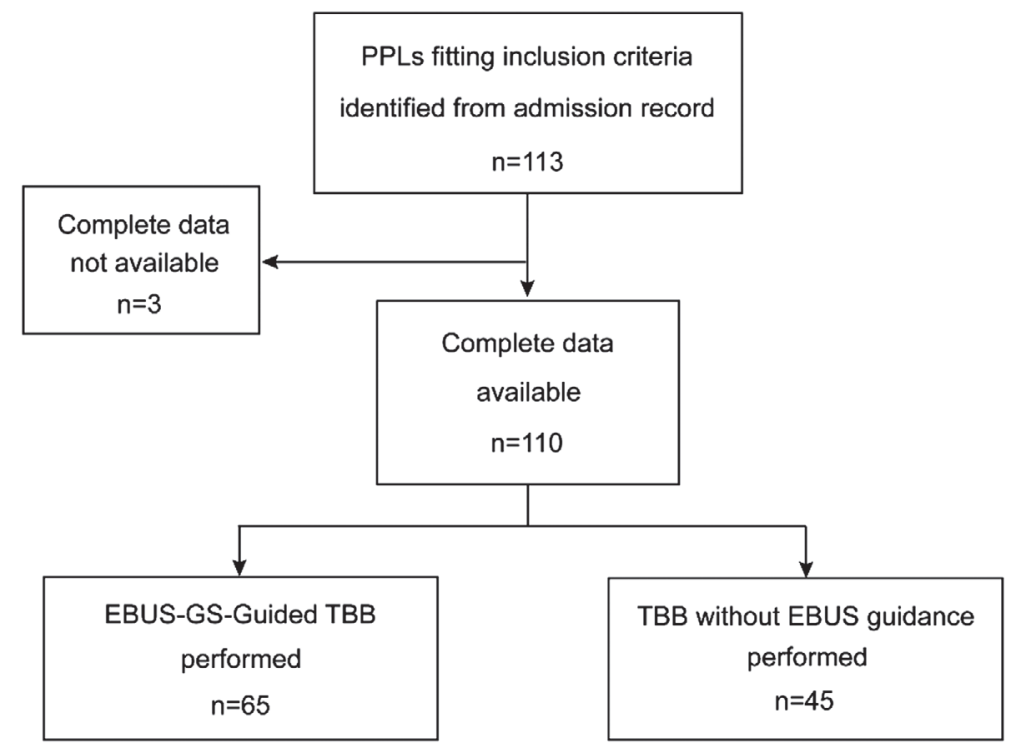

Figure 1. Description and categorization of lesions included in the study.

Table 1. Characteristics of Target Lesions, Nagasaki University Hospital

\begin{tabular}{|c|c|c|c|c|c|c|c|}
\hline \multirow[t]{2}{*}{ Characteristics } & \multicolumn{2}{|c|}{$\begin{array}{c}\text { Total } \\
(\mathrm{n}=110)\end{array}$} & \multicolumn{2}{|c|}{$\begin{array}{l}\text { TBB without EBUS guidance } \\
\qquad(\mathrm{n}=45)\end{array}$} & \multicolumn{2}{|c|}{$\begin{array}{l}\text { EBUS-GS-guided TBB } \\
\qquad(\mathrm{n}=65)\end{array}$} & \multirow[t]{2}{*}{$\mathrm{p}$ value ${ }^{\#}$} \\
\hline & N/Median & $\% / I Q R *$ & N/Median & $\% / \mathrm{IQR}$ & N/Median & $\% / \mathrm{IQR}$ & \\
\hline \multicolumn{8}{|l|}{ Sex } \\
\hline Female & 41 & 37.3 & 16 & 35.6 & 25 & 38.5 & \multirow{2}{*}{0.1} \\
\hline Male & 69 & 62.7 & 29 & 64.4 & 40 & 61.5 & \\
\hline Age (year) & 70.5 & 18 & 68 & 19 & 74 & 18 & $0.08^{\S}$ \\
\hline \multicolumn{8}{|l|}{ Size of lesions (mm) } \\
\hline$<20$ & 38 & 34.6 & 12 & 26.7 & 26 & 40.0 & \multirow{2}{*}{0.1} \\
\hline$\geq 20$ & 72 & 65.5 & 33 & 73.3 & 39 & 60.0 & \\
\hline \multicolumn{8}{|l|}{ Location of lesions } \\
\hline Upper/middle lobe & 72 & 65.5 & 27 & 60.0 & 45 & 69.2 & \multirow{2}{*}{0.3} \\
\hline Lower lobe & 38 & 34.5 & 18 & 40.0 & 20 & 30.8 & \\
\hline \multicolumn{8}{|l|}{ Diagnosis } \\
\hline Malignant lesions & 80 & 72.7 & 30 & 66.7 & 50 & 76.9 & \multirow{2}{*}{0.2} \\
\hline Benign lesions & 30 & 27.3 & 15 & 33.3 & 15 & 23.1 & \\
\hline
\end{tabular}

all patients before bronchoscopic procedures.

\section{Results}

113 PPLs met our inclusion criteria; 3 lesions were excluded from the study due to lack of complete data. Overall, a total of 110 lesions detected in 102 patients were enrolled to the study (Fig. 1).

The basic characteristics of lesions are shown in Table 1: 69 lesions $(62.7 \%)$ were from males and the median age of patients was 70.5 years (interquartile range 18). Median size of the lesions was $24.7 \mathrm{~mm}$ (IQR 20.3); 80 (72.7\%) were malignant lesions including primary lung cancer (non small cell carcinoma, $n=66$; small cell carcinoma, $n=3$; other $/$ not classified $n=5)$ and metastatic lung cancer $(n=6)$. Benign le- sions included infectious lung diseases $(n=13)$, inflammatory lung diseases $(n=8)$, and non-specific changes $(n=9)$.

Among all 110 lesions, EBUS-GS-guided TBB was performed on $65(59.1 \%)$ lesions while TBB without EBUS guidance was performed on $45(40.9 \%)$ lesions. All TBBs were performed under x-ray fluoroscopy. Of all EBUS examined lesions, $53(81.5 \%)$ were visualized by EBUS. The basic characteristics of each group are also shown in Table 1. Characteristics were similar between the two groups. Median size of the lesions in EBUS-GS group tended to be smaller than that in non-EBUS group although the difference was not statistically significant $(23.5 \mathrm{~mm}$ vs $29.5 \mathrm{~mm}$, $\mathrm{p}=0.08$ ). Major complications related to the procedure were observed in one case from EBUS-GS group (pneumothorax, $\mathrm{n}=1$ ) and in another case from non-EBUS group (pneumo- 
Table 2. Diagnostic Yield Ratios (95\% Confidence Intervals) of EBUS-GS-guided Bronchoscopy Compared to Conventional Bronchoscopy

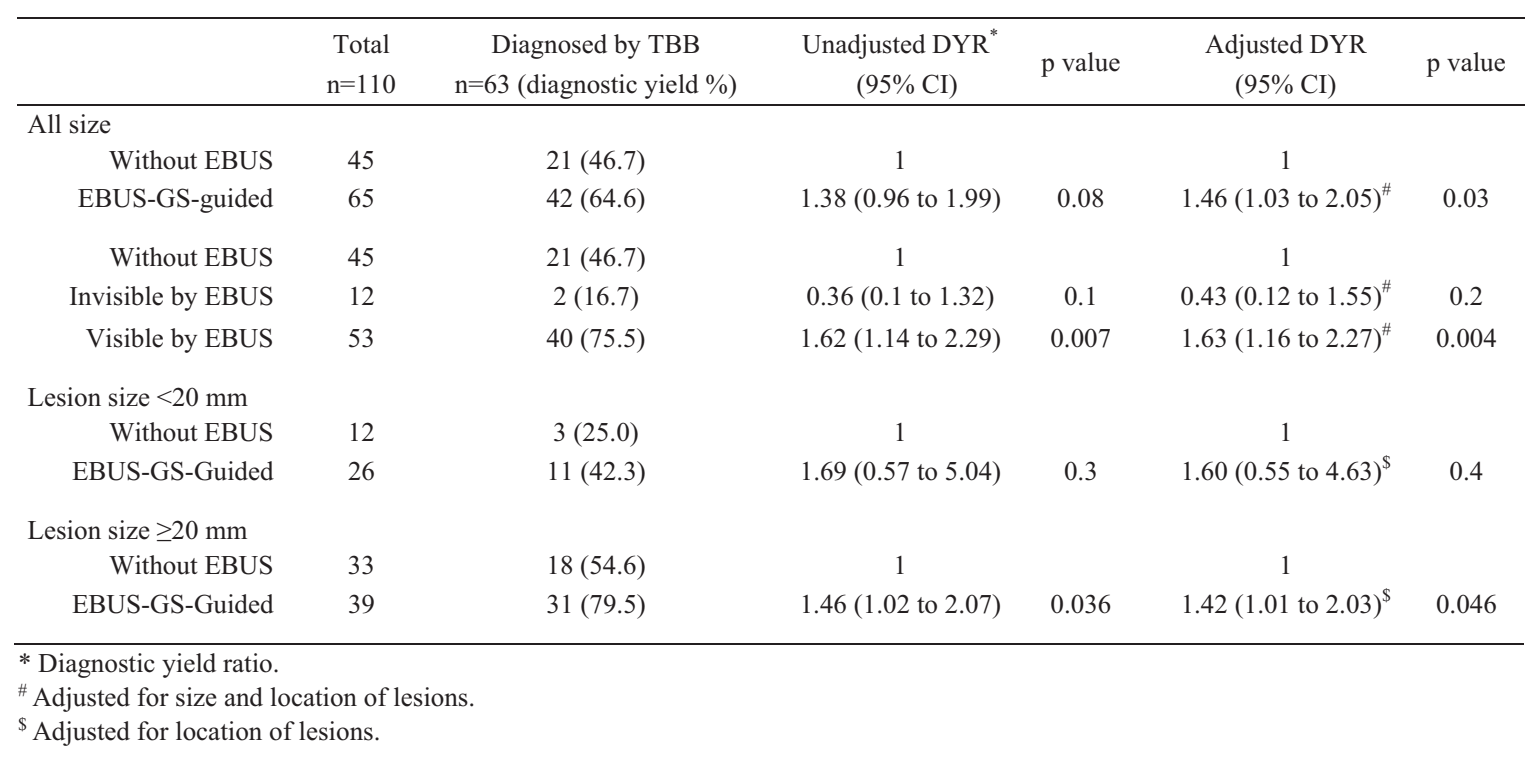

thorax, $\mathrm{n}=1$ ).

Diagnostic yields of EBUS-GS-guided TBB and TBB without EBUS guidance were $64.6 \%$ and $46.7 \%$, respectively (Table 2); $73.8 \%$ (31/42) of diagnoses in EBUS-GS group and $61.9 \%(13 / 21)$ of those in non-EBUS group were yielded by histopathology and the rest were yielded by cytology or washing culture. The diagnostic yield ratio of EBUS-GS group compared to non-EBUS group was 1.38 (95\% confidence interval 0.96 to 1.99 ). Adjusting for the size and location of lesions, the diagnostic yield in EBUSGS group was 1.46 (1.03 to 2.05) times higher than nonEBUS group. When the lesion was visualized by EBUS, the yield was 1.63 (1.16 to 2.27 ) times higher than the conventional procedure.

We also performed a subgroup analysis to evaluate the diagnostic effect of EBUS-GS in PPLs according to size of lesion. The diagnostic yield of EBUS-GS-guided TBB was higher than that of the conventional method in lesions of $\geq 20 \mathrm{~mm}$ (adjusted diagnostic yield ratio 1.42, 1.01 to 2.03). EBUS-GS also tended to perform better than the conventional method in lesions of $<20 \mathrm{~mm}$ but statistical support was low (1.60, 0.55 to 4.63$)$.

CT-NB or video-assisted thoracoscopic surgery for the purpose of diagnosis were less frequently performed in EBUS-GS group (10 of 65 lesions; 15.4\%) than in nonEBUS group (14 of 45 lesions; 31.1\%; $\mathrm{p}=0.05$, data not shown).

\section{Discussion}

EBUS-guided TBB using a guide sheath (GS) has been developed to overcome the limitations of conventional TBB procedures $(8,9)$. Recent studies have reported that EBUSGS guidance achieves a high diagnostic yield especially on small PPLs (58-79\%) (3, 8-12). However, these findings were based only on data for EBUS-GS guided procedure without comparing them with other procedures including $\mathrm{X}$ ray fluoroscopy-guided TBB. Our result indicates that the diagnostic yield of EBUS-GS-guided TBB on PPLs is 1.46 times higher than that of TBB without EBUS guidance. This is the first study to report a significant advantage of EBUSGS guidance over without EBUS guidance.

In the present study, $81.5 \%$ of lesions were visualized on EBUS which was comparable with other studies (75$93 \%)(3,4,8-11)$. Huang et al (7) showed that lesion size is a determining factor for the visibility of PPLs. This was also observed in our study (the visibility of nodules of $<20 \mathrm{~mm}$ diameter was $69.2 \%$; not shown in results). When the lesion was visualized by EBUS, the diagnostic yield of EBUS-GS reached $75.5 \%$, which was 1.63 times higher than fluoroscopy adjusting for size and location of lesions. On the other hand, this advantage was not observed on EBUS-invisible lesions. Specimen collections from EBUS-invisible lesions can be difficult even through GS. The present observation suggests that the diagnostic yield of EBUS-GS may be determined by the visibility of target PPL.

The present subgroup analysis showed that the diagnostic yield of EBUS-GS guidance was higher than fluoroscopic guidance for lesions of $\geq 20 \mathrm{~mm}$ diameter. EBUS-GS tended to perform better than fluoroscopic guidance also for small nodules of $<20 \mathrm{~mm}$, but the statistical support was low. The advantage may be smaller in small lesions because the probe position (i.e., within or adjacent to the PPL) determines the yield of EBUS-GS $(7,10)$. Our diagnostic yield of EBUS-GS-guided TBB on small nodules of $<20 \mathrm{~mm}$ (11/ $26=42.3 \%$ ) was relatively lower than those of other reports $(58-79 \%)(8-11)$ and in $55.6 \%$ of our visible small nodules $(10 / 18)$, the EBUS probe was positioned adjacent to the lesion. The yield on small nodules in which the probe position was adjacent to the lesion (5 of 10 nodules; $50.0 \%$ ) 
was lower than on nodules in which the probe position was within the lesion (5 of 8 nodules; $62.5 \%$ ). This might have weakened the diagnostic yield in our study. Due to the small sample size it would be premature to reach a conclusion about the effect of EBUS-GS on small nodules of $<20 \mathrm{~mm}$ from our findings. A larger prospective study, particularly for small lesions, is needed.

In the present study, no major complication was observed in EBUS-GS group except for one case of mild pneumothorax. Also, due to its high diagnostic yield, CT-NB and surgical biopsies were less frequently performed in EBUS-GS group. These findings support previous reports which have shown that EBUS-GS is a safe and beneficial procedure for patients with PPLs (8-11).

Limitations of this study arise from the nature of single centered retrospective design. EBUS-GS-guided TBBs were performed for all PPLs after the introduction of EBUS system. This may have resulted in slightly smaller lesion size in our EBUS-GS group. We therefore included the size of lesion in the final multiple regression models. Also characteristics of patients and our operators may have changed over the 6-year study period. However, roles of our department in the local health care system have been consistent over the study period, and all operators have been trained as chest physicians for more than 10 years. We believe that these factors had minimal effects on our findings. Another limitation was that the study did not show the effect of EBUS-GS-guidance over EBUS-alone procedure. Randomized controlled trials may be considered but it is natural that using GS with EBUS improves the diagnostic yield.

Lung cancer is the leading cause of cancer death in many developed countries including Japan (14) and United States (15). Recently, an early histological diagnosis is recognized as crucial for lung cancer management as therapeutic options become more specific in accordance with the findings of histological and genetic examinations $(16,17)$. To achieve high diagnostic yield on pulmonary lesions, new techniques have been developed such as CT fluoroscopicguided bronchoscopy (18), virtual navigation bronchoscopy (19), electromagnetic navigation bronchoscopy (20), and the combination of EBUS-guided TBB and positron emission tomography (21). However, these procedures require the introduction of costly instruments. We have clearly demonstrated that EBUS-GS, which can be easily adapted to the conventional bronchoscopic procedure, is a safe and beneficial method to collect samples from PPLs. EBUS-GSguided TBB, if available, should be the first choice procedure for the diagnosis of PPLs.

\section{The authors state that they have no Conflict of Interest (COI).}

\section{Acknowledgement}

First, we thank all physicians and other staff of the Department of Clinical Medicine, Institute of Tropical Medicine, Nagasaki University for their assistance during the study. We also thank Dr. Hirhoshi Nakaoka and Dr. Wolf-Peter Schmidt, who contributed to improve the manuscript.

Funding/Support: This research was supported in part by the Institute of Tropical Medicine, Nagasaki University, Japan. The funder had no role in study design, data collection and analysis, decision to publish, or preparation of the manuscript.

\section{References}

1. Schreiber G, McCrory DC. Performance characteristics of different modalities for diagnosis of suspected lung cancer: summary of published evidence. Chest 123(1 Suppl): 115S-128S, 2003.

2. Shinagawa N, Yamazaki K, Onodera Y, et al. CT-guided transbronchial biopsy using an ultrathin bronchoscope with virtual bronchoscopic navigation. Chest 125: 1138-1143, 2004.

3. Herth FJ, Eberhardt R, Becker HD, Ernst A. Endobronchial ultrasound-guided transbronchial lung biopsy in fluoroscopically invisible solitary pulmonary nodules: a prospective trial. Chest 129: 147-150, 2006.

4. Herth FJ, Ernst A, Becker HD. Endobronchial ultrasound-guided transbronchial lung biopsy in solitary pulmonary nodules and peripheral lesions. Eur Respir J 20: 972-974, 2002.

5. Paone G, Nicastri E, Lucantoni G, et al. Endobronchial ultrasound-driven biopsy in the diagnosis of peripheral lung lesions. Chest 128: 3551-3557, 2005.

6. Dooms CA, Verbeken EK, Becker HD, Demedts MG, Vansteenkiste JF. Endobronchial ultrasonography in bronchoscopic occult pulmonary lesions. J Thorac Oncol 2: 121-124, 2007.

7. Huang CT, Ho CC, Tsai YJ, Yu CJ, Yang PC. Factors influencing visibility and diagnostic yield of transbronchial biopsy using endobronchial ultrasound in peripheral pulmonary lesions. Respirology 14: 859-864, 2009.

8. Kurimoto N, Miyazawa T, Okimasa S, et al. Endobronchial ultrasonography using a guide sheath increases the ability to diagnose peripheral pulmonary lesions endoscopically. Chest 126: 959-965, 2004.

9. Kikuchi E, Yamazaki K, Sukoh N, et al. Endobronchial ultrasonography with guide-sheath for peripheral pulmonary lesions. Eur Respir J 24: 533-537, 2004.

10. Yamada N, Yamazaki K, Kurimoto N, et al. Factors related to diagnostic yield of transbronchial biopsy using endobronchial ultrasonography with a guide sheath in small peripheral pulmonary lesions. Chest 132: 603-608, 2007.

11. Yoshikawa M, Sukoh N, Yamazaki K, et al. Diagnostic value of endobronchial ultrasonography with a guide sheath for peripheral pulmonary lesions without X-ray fluoroscopy. Chest 131: 17881793, 2007.

12. Eberhardt R, Ernst A, Herth FJ. Ultrasound-guided transbronchial biopsy of solitary pulmonary nodules less than $20 \mathrm{~mm}$. Eur Respir J 34: 1284-1287, 2009.

13. McNutt LA, Wu C, Xue X, Hafner JP. Estimating the relative risk in cohort studies and clinical trials of common outcomes. Am J Epidemiol 157: 940-943, 2003.

14. Cancer Statistics in Japan 2009. Foundation for Promotion of Cancer Research, Tokyo, 2009.

15. Jemal A, Thun MJ, Ries LA, et al. Annual report to the nation on the status of cancer, 1975-2005, featuring trends in lung cancer, tobacco use, and tobacco control. J Natl Cancer Inst 100: 16721694, 2008.

16. Tiseo M, Bartolotti M, Gelsomino F, Ardizzoni A. First-line treatment in advanced non-small-cell lung cancer: the emerging role of the histologic subtype. Expert Rev Anticancer Ther 9: 425-435, 2009.

17. Dubey S, Powell CA. Update in lung cancer 2008. Am J Respir Crit Care Med 179: 860-868, 2009.

18. Ost D, Shah R, Anasco E, et al. A randomized trial of $\mathrm{CT}$ 
fluoroscopic-guided bronchoscopy vs conventional bronchoscopy in patients with suspected lung cancer. Chest 134: 507-513, 2008.

19. Asahina H, Yamazaki K, Onodera Y, et al. Transbronchial biopsy using endobronchial ultrasonography with a guide sheath and virtual bronchoscopic navigation. Chest 128: 1761-1765, 2005.

20. Eberhardt R, Anantham D, Ernst A, Feller-Kopman D, Herth F. Multimodality bronchoscopic diagnosis of peripheral lung lesions: a randomized controlled trial. Am J Respir Crit Care Med 176: 36-41, 2007.

21. Mizugaki H, Shinagawa N, Kanegae K, et al. Combining transbronchial biopsy using endobronchial ultrasonography with a guide sheath and positron emission tomography for the diagnosis of small peripheral pulmonary lesions. Lung Cancer 68: 211-215, 2010.

(C) 2012 The Japanese Society of Internal Medicine http://www.naika.or.jp/imindex.html 